General Mathematics Vol. 28, No. 1 (2020), 41-57

DOI: $10.2478 / \mathrm{gm}-2020-0004$

Sciendo

\title{
Common fixed points under contractive conditions of integral type ${ }^{1}$
}

\author{
Hakima Bouhadjera
}

\begin{abstract}
In this paper, we give some common fixed point theorems for a class of occasionally weakly compatible mappings satisfying contractive conditions of integral type. Our results generalize a host of previously theorems. We also present some illustrative examples which support our main results and show the applicability and validity of these results.
\end{abstract}

2010 Mathematics Subject Classification: 47H10, 54H25.

Key words and phrases: Weakly compatible mappings, occasionally weakly compatible mappings, contractive conditions, integral type, common fixed point theorems.

\footnotetext{
${ }^{1}$ Received 10 October, 2019

Accepted for publication (in revised form) 25 May, 2020
} 


\section{Introduction and Preliminaries}

In 1982, Sessa [19] defined the concept of weakly commuting mappings as a generalization of commuting mappings.

Further, Jungck generalized this idea to compatible mappings [4].

In 1993, the same author with Murthy and Cho [6] gave another generalization of commuting mappings by introducing the notion of compatible mappings of type $(A)$.

In [12], Pathak and Khan made a generalization of compatible mappings of type $(A)$ by giving the concept of compatible mappings of type $(B)$.

The first author with Cho, Kang and Lee [10] introduced another type of compatibility called compatibility of type $(P)$.

In 1998, Pathak et al. [11] introduced another extension of compatible mappings of type $(A)$ by giving the notion of compatible mappings of type $(C)$, and they gave an example to show that compatible mappings of type $(C)$ need not be compatible, nor compatible of type $(A)$ and type $(B)$.

In [5], Jungck generalized all the above concepts to weakly compatible mappings.

Recently, Al-Thagafi and Shahzad [1] introduced a generalization of weakly compatible mappings by giving the notion of occasionally weakly compatible mappings.

Let $(\mathcal{X}, d)$ be a metric space and let $f$ and $g$ be two self-mappings of $\mathcal{X}$.

Definition 1 ([19]) Two self-mappings $f$ and $g$ of a metric space $(\mathcal{X}, d)$ are said to be weakly commuting if

$$
d(f g x, g f x) \leq d(g x, f x)
$$

for all $x \in \mathcal{X}$.

Definition 2 ([4],[6],[12],[11],[10]) Two self-mappings $f$ and $g$ of a metric space $(\mathcal{X}, d)$ are said to be

1. compatible if

$$
\lim _{n \rightarrow \infty} d\left(f g x_{n}, g f x_{n}\right)=0
$$


2. compatible of type $(A)$ if

$$
\lim _{n \rightarrow \infty} d\left(f g x_{n}, g^{2} x_{n}\right)=0 \text { and } \lim _{n \rightarrow \infty} d\left(g f x_{n}, f^{2} x_{n}\right)=0,
$$

3. compatible of type $(B)$ if

$$
\begin{aligned}
\lim _{n \rightarrow \infty} d\left(f g x_{n}, g^{2} x_{n}\right) \leq & \frac{1}{2}\left[\lim _{n \rightarrow \infty} d\left(f g x_{n}, f t\right)+\lim _{n \rightarrow \infty} d\left(f t, f^{2} x_{n}\right)\right] \\
& \text { and } \\
\lim _{n \rightarrow \infty} d\left(g f x_{n}, f^{2} x_{n}\right) \leq & \frac{1}{2}\left[\lim _{n \rightarrow \infty} d\left(g f x_{n}, g t\right)+\lim _{n \rightarrow \infty} d\left(g t, g^{2} x_{n}\right)\right],
\end{aligned}
$$

4. compatible of type $(C)$ if

$$
\begin{aligned}
\lim _{n \rightarrow \infty} d\left(f g x_{n}, g^{2} x_{n}\right) \leq & \frac{1}{3}\left[\lim _{n \rightarrow \infty} d\left(f g x_{n}, f t\right)+\lim _{n \rightarrow \infty} d\left(f t, f^{2} x_{n}\right)\right. \\
& \left.+\lim _{n \rightarrow \infty} d\left(f t, g^{2} x_{n}\right)\right] \\
& \text { and } \\
\lim _{n \rightarrow \infty} d\left(g f x_{n}, f^{2} x_{n}\right) \leq & \frac{1}{3}\left[\lim _{n \rightarrow \infty} d\left(g f x_{n}, g t\right)+\lim _{n \rightarrow \infty} d\left(g t, g^{2} x_{n}\right)\right. \\
& \left.+\lim _{n \rightarrow \infty} d\left(g t, f^{2} x_{n}\right)\right],
\end{aligned}
$$

5. compatible of type $(P)$ if

$$
\lim _{n \rightarrow \infty} d\left(f^{2} x_{n}, g^{2} x_{n}\right)=0
$$

whenever $\left\{x_{n}\right\}$ is a sequence in $\mathcal{X}$ such that $\lim _{n \rightarrow \infty} f x_{n}=\lim _{n \rightarrow \infty} g x_{n}=t$ for some $t \in \mathcal{X}$.

Definition 3 ([5]) Two self-mappings $f$ and $g$ of a metric space $(\mathcal{X}, d)$ are said to be weakly compatible if they commute at coincidence points; that is, $f x=g x$, then, $f g x=g f x$.

Definition 4 ([千]) Let $\mathcal{X}$ be a set and let $f, g$ self-mappings of $\mathcal{X}$. A point $x$ in $\mathcal{X}$ is called a coincidence point of $f$ and $g$ if and only if $f x=g x$.

Definition 5 ([1]) Two self-mappings $f$ and $g$ of a set $\mathcal{X}$ are occasionally weakly compatible (owc) if and only if there is a point $x$ in $\mathcal{X}$ which is a coincidence point of $f$ and $g$ at which $f$ and $g$ commute. 
Definition 6 Let $\mathcal{X}$ be a nonempty set. A symmetric on $\mathcal{X}$ is a mapping $d: \mathcal{X} \times \mathcal{X} \rightarrow[0, \infty)$ such that

1. $d(x, y)=0$ if and only if $x=y$ and

2. $d(x, y)=d(y, x)$ for $x, y \in \mathcal{X}$.

Now, let $\mathcal{F}$ be the set of all functions $F: \mathbb{R}_{+}^{6} \rightarrow \mathbb{R}_{+}$satisfying condition:

$\left(F_{1}\right) \int_{0}^{F(u, u, 0,0, u, u)} \varphi(t) d t>0$; for all $u>0$ where $\varphi: \mathbb{R}_{+} \rightarrow \mathbb{R}$ is a Lebesgue-integrable mapping which is summable.

Example 1 Let $F\left(t_{1}, t_{2}, t_{3}, t_{4}, t_{5}, t_{6}\right)=t_{1}-c \max \left\{t_{2}, t_{3}, t_{4}, t_{5}, t_{6}\right\}$, where $c \in$ $(0,1)$, and $\varphi(t)=2 t$ for all $t$ in $\mathbb{R}_{+}$. Then

$$
\left(F_{1}\right) \int_{0}^{F(u, u, 0,0, u, u)} \varphi(t) d t=\int_{0}^{u(1-c)} 2 t d t=u^{2}(1-c)^{2}>0 \text {, for all } u>0 .
$$

\section{Main Results}

In this section, we prove some common fixed point theorems for pairs of selfmappings defined on a nonempty set endowed with a symmetric. Our results contain many theorems of single-self-mappings in a metric space satisfying a contractive condition (resp. a contractive condition of integral type) as special cases.

We start by the next result.

Theorem 1 Let $\mathcal{X}$ be a nonempty set with a symmetric $d$. Let $f, g, h$ and $k$ be self-mappings of $\mathcal{X}$. Suppose there exists $F \in \mathcal{F}$ such that

$$
\int_{0}^{F(d(f x, g y), d(h x, k y), d(h x, f x), d(k y, g y), d(h x, g y), d(k y, f x))} \varphi(t) d t \leq 0
$$

for all $x y$ in $\mathcal{X}$. If $f$ and $h$ as well as $g$ and $k$ are owc, then $f, g$, $h$ and $k$ have a unique common fixed point.

Proof. Since $f$ and $h$ as well as $g$ and $k$ are owc, there exist points $u$ and $v$ in $\mathcal{X}$ such that $f u=h u, f h u=h f u$ and $g v=k v, g k v=k g v$. 
First, we claim that $f u=g v$. By inequality (1) we get

$$
\begin{aligned}
& \int_{0}^{F(d(f u, g v), d(h u, k v), d(h u, f u), d(k v, g v), d(h u, g v), d(k v, f u))} \varphi(t) d t \\
= & \int_{0}^{F(d(f u, g v), d(f u, g v), 0,0, d(f u, g v), d(g v, f u))} \varphi(t) d t \leq 0
\end{aligned}
$$

which contradicts $\left(F_{1}\right)$. Hence, $f u=g v$.

Suppose that $f^{2} u \neq f u$, then, from (1),

$$
\begin{aligned}
& \int_{0}^{F\left(d\left(f^{2} u, g v\right), d(h f u, k v), d\left(h f u, f^{2} u\right), d(k v, g v), d(h f u, g v), d\left(k v, f^{2} u\right)\right)} \varphi(t) d t \\
= & \int_{0}^{F\left(d\left(f^{2} u, f u\right), d\left(f^{2} u, f u\right), 0,0, d\left(f^{2} u, f u\right), d\left(f u, f^{2} u\right)\right)} \varphi(t) d t \leq 0
\end{aligned}
$$

which contradicts $\left(F_{1}\right)$. Therefore $f^{2} u=f u$.

Similarly, $g^{2} v=g v$. Put $f u=g v=z$, then, $f z=h z=g z=k z=z$.

If there is another point $t \neq z$ such that $f t=h t=g t=k t=t$, then, using (1) it follows that $t=z$.

Since every contractive condition of integral type includes a non-involving integrals contractive condition, main results of [2], ([14]-[17]) are special cases of Theorem 1.

Corollary 1 Let $\mathcal{X}$ be a nonempty set with a symmetric $d$. Let $f$ and $h$ be owc mappings of $\mathcal{X}$. Suppose there exists $F \in \mathcal{F}$ such that

$$
\int_{0}^{F(d(f x, f y), d(h x, h y), d(h x, f x), d(h y, f y), d(h x, f y), d(h y, f x))} \varphi(t) d t \leq 0
$$

for all $x y$ in $\mathcal{X}$. Then, $f$ and $h$ have a unique common fixed point.

Corollary 2 Let $d$ be a symmetric for the nonempty set $\mathcal{X}$, and let $f, g$ and $h$ be mappings from $\mathcal{X}$ into itself such that $f$ and $h$ as well as $g$ and $h$ are owc. Suppose there exists $F \in \mathcal{F}$ such that

$$
\int_{0}^{F(d(f x, g y), d(h x, h y), d(h x, f x), d(h y, g y), d(h x, g y), d(h y, f x))} \varphi(t) d t \leq 0
$$

for all $x y$ in $\mathcal{X}$. Then, $f, g$ and $h$ have a unique common fixed point. 
For $\varphi(t)=1$ in Theorem 1, we obtain the following corollary, which improves some results especially Corollary 3.1 of [13] and main results of [2] and ([14]-[17]).

Corollary 3 Let $d$ be a symmetric for the nonempty set $\mathcal{X}$, and let $f, g, h$ and $k$ be self-mappings of $\mathcal{X}$. Suppose there exists $F: \mathbb{R}_{+}^{6} \rightarrow \mathbb{R}$ satisfies the condition: $F(u, u, 0,0, u, u)>0$ for all $u>0$ and

$$
F(d(f x, g y), d(h x, k y), d(h x, f x), d(k y, g y), d(h x, g y), d(k y, f x)) \leq 0
$$

for all $x, y$ in $\mathcal{X}$. If $f$ and $h$ as well as $g$ and $k$ are owc, then, $f, g, h$ and $k$ have a unique common fixed point.

If we let $F\left(t_{1}, t_{2}, t_{3}, t_{4}, t_{5}, t_{6}\right)=t_{1}-c \max \left\{t_{2}, t_{3}, t_{4} t_{5}, t_{6}\right\}$ in Corollary 3.4 , where $0<c<1$, then we have the following result which extends some results especially Corollary 3.2 of [13].

Corollary 4 Let $d$ be a symmetric for the nonempty set $\mathcal{X}$, and let $f, g, h$ and $k$ be self-mappings of $\mathcal{X}$ which satisfy the next inequality

$$
d(f x, g y) \leq c \max \{d(h x, k y), d(h x, f x), d(k y, g y) d(h x, g y), d(k y, f x)\}
$$

for all $x, y$ in $\mathcal{X}$, where $0<c<1$. Suppose that $f$ and $h$ as well as $g$ and $k$ are owc, then, $f, g, h$ and $k$ have a unique common fixed point.

Remark 1 1. Theorem 1 generalizes the result of Pathak et al. [13], since the four mappings are only owc and $F$ satisfies only property $\left(F_{1}\right)$.

2. If we put in inequality (1)

$$
\begin{aligned}
& F(d(f x, g y), d(h x, k y), d(h x, f x), d(k y, g y), d(h x, g y), d(k y, f x)) \\
= & d(f x, g y)-c \max \{d(h x, k y), d(h x, f x), d(k y, g y), \\
& \left.\frac{1}{2}(d(h x, g y)+d(k y, f x))\right\}
\end{aligned}
$$

where $c \in[0,1)$, we get

$$
\int_{0}^{d(f x, g y)-c \max \left\{d(h x, k y), d(h x, f x), d(k y, g y), \frac{1}{2}(d(h x, g y)+d(k y, f x))\right\}} \varphi(t) d t \leq 0
$$


which implies that

$$
\begin{aligned}
& \int_{0}^{d(f x, g y)} \varphi(t) d t \\
\leq & \int_{0}^{c \max \left\{d(h x, k y), d(h x, f x), d(k y, g y), \frac{1}{2}(d(h x, g y)+d(k y, f x))\right\}} \varphi(t) d t \\
< & \int_{0}^{\max \{d(h x, k y), d(h x, f x), d(k y, g y), d(h x, g y), d(k y, f x)\}} \varphi(t) d t
\end{aligned}
$$

i.e., Theorem 1 and Corollary 1 of [7] are special cases of our result if $\varphi(t)=1$.

3. If we put $f=g, h=k$ and

$$
\begin{aligned}
& F(d(f x, f y), d(h x, h y), d(h x, f x), d(h y, f y), d(h x, f y), d(h y, f x)) \\
= & d(f x, f y)-c d(h x, h y)
\end{aligned}
$$

with $c \in[0,1)$ we get a generalization of Theorem 2.1 of Kumar et al. [9].

4. If we let $f=g, h=k=\mathcal{I}_{\mathcal{X}}$ (the identity mapping on $\mathcal{X}$ ) and

$$
\begin{aligned}
& F(d(f x, f y), d(x, y), d(x, f x), d(y, f y), d(x, f y), d(y, f x)) \\
= & d(f x, f y)-c \max \left\{d(x, y), d(x, f x), d(y, f y), \frac{1}{2}(d(x, f y)+d(y, f x))\right\}
\end{aligned}
$$

or

$$
\begin{aligned}
& F(d(f x, f y), d(x, y), d(x, f x), d(y, f y), d(x, f y), d(y, f x)) \\
= & d(f x, f y)-c \max \{d(x, y), d(x, f x), d(y, f y), d(x, f y), d(y, f x)\}
\end{aligned}
$$

where $c \in[0,1)$, we get generalizations of Theorems 2 and 4 of Rhoades [18].

5. Putting $f=g, h=k=\mathcal{I}_{\mathcal{X}}, c \in(0,1)$ and

$$
\begin{aligned}
& F(d(f x, f y), d(x, y), d(x, f x), d(y, f y), d(x, f y), d(y, f x)) \\
= & d(f x, f y)-c d(x, y)
\end{aligned}
$$

we obtain a generalization of Theorem 2.1 of Branciari [3]. 
6. If we let $f=g, h=k, c \in[0,1)$ and

$$
\begin{aligned}
& F(d(f x, f y), d(h x, h y), d(h x, f x), d(h y, f y), d(h x, f y), d(h y, f x)) \\
= & d(f x, f y)-c \max \{d(h x, h y), d(h x, f x), d(h y, f y), \\
& \left.\frac{1}{2}(d(h x, f y)+d(h y, f x))\right\}
\end{aligned}
$$

we get a generalization of Theorem 1 of Kohli and Vashistha [8] and if we let

$$
\begin{aligned}
& F(d(f x, f y), d(h x, h y), d(h x, f x), d(h y, f y), d(h x, f y), d(h y, f x)) \\
= & d(f x, f y)-c \max \{d(h x, h y), d(h x, f x), d(h y, f y), \\
& \left.\frac{1}{2} d(h x, f y), \frac{1}{2} d(h y, f x)\right\}
\end{aligned}
$$

we obtain a generalization of Theorem 2 of [8].

7. If $f=g, h=k=\mathcal{I}_{\mathcal{X}}, c \in[0,1)$ and

$$
\begin{aligned}
& F(d(f x, f y), d(x, y), d(x, f x), d(y, f y), d(x, f y), d(y, f x)) \\
& =d(f x, f y)-c \max \left\{d(x, y), d(x, f x), d(y, f y), \frac{1}{2}(d(x, f y)+d(y, f x))\right\} \\
& \text { we get a generalization of Corollary } 1 \text { of [8]. }
\end{aligned}
$$

8. Letting $f=g, h=k=\mathcal{I}_{\mathcal{X}}, c \in[0,1)$ and

$$
\begin{aligned}
& F(d(f x, f y), d(x, y), d(x, f x), d(y, f y), d(x, f y), d(y, f x)) \\
= & d(f x, f y)-c \max \left\{d(x, y), d(x, f x), d(y, f y), \frac{1}{2} d(x, f y), \frac{1}{2} d(y, f x)\right\}
\end{aligned}
$$

we get a generalization of Corollary 2 of [8].

9. When $f=g, h=k=\mathcal{I}_{\mathcal{X}}, c \in[0,1)$ and

$$
\begin{aligned}
& F(d(f x, f y), d(x, y), d(x, f x), d(y, f y), d(x, f y), d(y, f x)) \\
= & d(f x, f y)-c d(x, y)
\end{aligned}
$$

we obtain a generalization of Corollary 3 of [8]. 
10. Also, Theorems 3 and 4 of [8] are special cases of our first result.

Example 2 Let $\mathcal{X}=[0, \infty)$ endowed with the symmetric $d(x, y)=\exp ^{|x-y|}-1$. Define

$$
\begin{gathered}
f x=\left\{\begin{array}{ll}
\frac{1}{2} & \text { if } x \in[0,1) \\
1 & \text { if } x \in[1, \infty),
\end{array} \quad g y=\left\{\begin{array}{cc}
\frac{1}{4} & \text { if } y \in[0,1) \\
1 & \text { if } y \in[1, \infty),
\end{array}\right.\right. \\
h x=\left\{\begin{array}{ll}
2 & \text { if } x \in[0,1) \\
\frac{1}{x^{2}} & \text { if } x \in[1, \infty),
\end{array} \quad k y= \begin{cases}3 & \text { if } y \in[0,1) \\
\frac{1}{y} & \text { if } y \in[1, \infty) .\end{cases} \right.
\end{gathered}
$$

Take $\varphi(t)=2 t$ and $F\left(t_{1}, t_{2}, t_{3}, t_{4}, t_{5}, t_{6}\right)=t_{1}-\frac{1}{2} t_{2}$, so, all conditions of Theorem 1 are satisfied and 1 is the unique common fixed point of mappings $f, g, h$ and $k$.

Now, we give our second result.

Theorem 2 Let $\mathcal{X}$ be a nonempty set with a symmetric $d$, let $f, g, h$ and $k$ be self-mappings of $\mathcal{X}$ such that $\int_{0}^{d(2)} \varphi(t) d t \leq \int_{0}^{\phi\left(\max \left\{d(h x, k y), d(f x, h x), d(g y, k y), \frac{1}{2}(d(f x, k y)+d(g y, h x))\right\}\right)} \varphi(t) d t$ where $\varphi: \mathbb{R}_{+} \rightarrow \mathbb{R}_{+}$is a Lebesgue-integrable mapping which is summable such that $\int_{0}^{\epsilon} \varphi(t) d t>0$ for each $\epsilon>0$ and $\phi: \mathbb{R}_{+} \rightarrow \mathbb{R}_{+}$with $\phi(t)<t$ for each $t>0$. If $f$ and $h$ as well as $g$ and $k$ are owc, then $f, g, h$ and $k$ have $a$ unique common fixed point.

\section{Proof.}

Existence: By hypothesis, there exist two elements $u$ and $v$ in $\mathcal{X}$ such that $f u=h u, f h u=h f u$ and $g v=k v, g k v=k g v$.

Suppose that $d(f u, g v)>0$, then, from (2) we get

$$
\begin{aligned}
& \int_{0}^{d(f u, g v)} \varphi(t) d t \\
\leq & \int_{0}^{\phi\left(\max \left\{d(h u, k v), d(f u, h u), d(g v, k v), \frac{1}{2}(d(f u, k v)+d(g v, h u))\right\}\right)} \varphi(t) d t \\
= & \int_{0}^{\phi(d(f u, g v))} \varphi(t) d t<\int_{0}^{d(f u, g v)} \varphi(t) d t
\end{aligned}
$$


a contradiction. Therefore $f u=g v$.

If $d\left(f^{2} u, f u\right)>0$, then, $(2)$ gives

$$
\begin{aligned}
& \int_{0}^{d\left(f^{2} u, f u\right)} \varphi(t) d t=\int_{0}^{d(f f u, g v)} \varphi(t) d t \\
\leq & \int_{0}^{\phi\left(\max \left\{d(h f u, k v), d\left(f^{2} u, h f u\right), d(g v, k v), \frac{1}{2}\left(d\left(f^{2} u, k v\right)+d(g v, h f u)\right)\right\}\right)} \varphi(t) d t \\
= & \int_{0}^{\phi\left(d\left(f^{2} u, f u\right)\right)} \varphi(t) d t<\int_{0}^{d\left(f^{2} u, f u\right)} \varphi(t) d t
\end{aligned}
$$

a contradiction. Therefore, $f^{2} u=f u$.

Similarly, $g^{2} v=g v$ and $f u=g v=z$ is a common fixed point of $f, g, h$ and $k$.

Uniqueness: follows immediately from inequality (2).

Remark 2 If we let $h=k$ in the above Theorem, we get a generalization of Corollary 3 of [r].

Example 3 Consider the set $\mathcal{X}=[0, \infty)$ with the symmetric $d(x, y)=\ln [\mid x-$ $y \mid+1]$. Define

$$
\begin{aligned}
& f x=\left\{\begin{array}{ll}
\frac{3}{4} & \text { if } x \in[0,1) \\
1 & \text { if } x \in[1, \infty),
\end{array} \quad g y= \begin{cases}\frac{1}{2} & \text { if } y \in[0,1) \\
1 & \text { if } y \in[1, \infty),\end{cases} \right. \\
& h x=\left\{\begin{array}{ll}
10 & \text { if } x \in[0,1) \\
\frac{1}{x} & \text { if } x \in[1, \infty),
\end{array} \quad k y= \begin{cases}5 & \text { if } y \in[0,1) \\
\frac{1}{y} & \text { if } y \in[1, \infty) .\end{cases} \right.
\end{aligned}
$$

Let $\varphi(t)=t$ and $\phi(t)=\frac{t}{2}$, so, all conditions of Theorem 2 are satisfied and 1 is the unique common fixed point of mappings $f, g, h$ and $k$.

The following Theorem is our third result.

Theorem 3 Let $\mathcal{X}$ be a nonempty set with a symmetric d, let $f$ and $h$ be owc self-mappings of $\mathcal{X}$ which satisfy

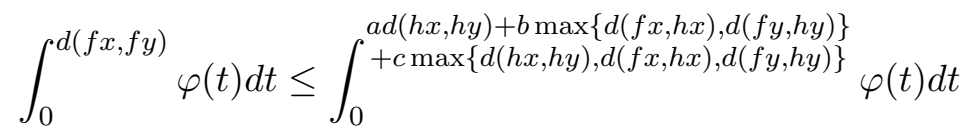


for all $x, y$ in $\mathcal{X}$, where $a, b, c>0, a+b+c \leq 1$ and $\varphi$ is as in Theorem 2. Then $f$ and $h$ have a unique common fixed point.

Proof. Since $f$ and $h$ are owc, then, there is an element $u \in \mathcal{X}$ such that $f u=h u$ and $f h u=h f u$.

We claim that $f^{2} u=h f u=f u$. If not, then, from (3)

$$
\begin{aligned}
& \int_{0}^{d\left(f^{2} u, f u\right)} \varphi(t) d t \leq \\
& \int_{0}^{a d(h f u, h u)+b \max \left\{d\left(f^{2} u, h f u\right), d(f u, h u)\right\}+c \max \{d(h f u, h u),} \dot{\left.d\left(f^{2} u, h f u\right), d(f u, h u)\right\}} \varphi(t) d t \\
= & \int_{0}^{(a+c) d\left(f^{2} u, f u\right)} \varphi(t) d t<\int_{0}^{d\left(f^{2} u, f u\right)} \varphi(t) d t
\end{aligned}
$$

a contradiction. Hence, $f f u=h f u=f u=z$.

Suppose that there exists another common fixed point $t \neq z$. Then, by (3) we obtain

$$
\begin{aligned}
& \int_{0}^{d(z, t)} \varphi(t) d t=\int_{0}^{d(f z, f t)} \varphi(t) d t \leq \\
& \int_{0}^{a d(h z, h t)+b \max \{d(f z, h z), d(f t, h t)\}+c \max \{d(h z, h t),} \varphi(t) d t \\
= & \int_{0}^{(a+c) d(z, h z), d(f t, h t)\}} \varphi(t) d t<\int_{0}^{d(z, t)} \varphi(t) d t
\end{aligned}
$$

a contradiction. Thus, $t=z$.

Remark 3 If we put $\varphi(t)=1$ in the above Theorem, we get a generalization of Theorem 2 of [ $[7]$.

Example 4 Endow $\mathcal{X}=[0, \infty)$ with the symmetric $d(x, y)=(x-y)^{2}$. Define two mappings $f$ and $h$ as follows:

$$
f x=\left\{\begin{array}{ll}
0 & \text { if } x \in[0,1) \\
1 & \text { if } x \in[1, \infty),
\end{array} \quad g y=\left\{\begin{array}{cl}
4 & \text { if } y \in[0,1) \\
\frac{1}{y} & \text { if } y \in[1, \infty) .
\end{array}\right.\right.
$$

Let $\varphi(t)=3 t^{2}, a=\frac{1}{2}$ and $b=c=\frac{1}{4}$, so, all conditions of Theorem 3 are satisfied and 1 is the unique common fixed point of mappings $f, g, h$ and $k$. 
Our fourth result is as follows:

Theorem 4 Let $\mathcal{X}$ be a nonempty set with a symmetric $d$, let $f, g, h$ and $k$ be self-mappings of $\mathcal{X}$ satisfying

(4) $\int_{0}^{d^{p}(f x, g y)} \varphi(t) d t<\int_{0}^{a d^{p}(h x, k y)+(1-a) \max \left\{\alpha d^{p}(f x, h x), \beta d^{p}(g y, k y),\right.} d^{\left.\frac{p}{2}(f x, h x) d^{\frac{p}{2}}(f x, k y), d^{\frac{p}{2}}(f x, k y) d^{\frac{p}{2}}(g y, h x)\right\}} \varphi(t) d t$

for all $x, y$ in $\mathcal{X}$, where $0<a, \alpha, \beta \leq 1, p \geq 1$ and $\varphi$ is as in Theorem 2. If $f$ and $h$ as well as $g$ and $k$ are owc, then $f, g$, $h$ and $k$ have a unique common fixed point.

Proof. By hypothesis, there exist two points $u$ and $v$ such that $f u=h u$, $g v=k v$ and $f h u=h f u, g k v=k g v$.

Suppose that $f u \neq g v$. Then, from (4),

$$
\begin{aligned}
& \int_{0}^{d^{p}(f u, g v)} \varphi(t) d t< \\
& \int_{0}^{a d^{p}(h u, k v)+(1-a) \max \left\{\alpha d^{p}(f u, h u), \beta d^{p}(g v, k v), d^{\frac{p}{2}}(f u, h u) d^{\frac{p}{2}}(f u, k v),\right.}, \quad d^{\left.\frac{p}{2}(f u, k v) d^{\frac{p}{2}}(g v, h u)\right\}} \\
= & \int_{0}^{d^{p}(f u, g v)} \varphi(t) d t
\end{aligned}
$$

a contradiction. Therefore $d(f u, g v)=0$, which implies that $f u=g v$.

If $g^{2} v \neq g v$. Then, from (4),

$$
\begin{aligned}
& \int_{0}^{d^{p}\left(g v, g^{2} v\right)} \varphi(t) d t=\int_{0}^{d^{p}\left(f u, g^{2} v\right)} \varphi(t) d t< \\
& \int_{0} a d^{p}(h u, k g v)+(1-a) \max \left\{\alpha d^{p}(f u, h u), \beta d^{p}\left(g^{2} v, k g v\right), d^{\frac{p}{2}}(f u, h u) d^{\frac{p}{2}}(f u, k g v),\right. \\
\left.d^{\frac{p}{2}}(f u, k g v) d^{\frac{p}{2}}\left(g^{2} v, h u\right)\right\} & \varphi(t) d t \\
= & \int_{0}^{d^{p}\left(g v, g^{2} v\right)} \varphi(t) d t
\end{aligned}
$$

which is a contradiction. Hence $g^{2} v=g v$.

Similarly, $f^{2} u=f u$ and $f u=g v$ is a common fixed point of $f, g, h$ and $k$.

From condition (4), it follows easily that $f u$ is unique. 
Remark 4 If $\varphi(t)=1$, we get a generalization of Theorem 3 of [7].

Example 5 Consider the set $\mathcal{X}=[0, \infty)$ with the symmetric $d(x, y)=(x-$ $y)^{2}$. Define

$$
\begin{gathered}
f x=g y=\left\{\begin{array}{cl}
\frac{3}{4} & \text { if } x, y \in[0,1) \\
1 & \text { if } x, y \in[1, \infty),
\end{array} \quad h x=\left\{\begin{array}{cc}
7 & \text { if } x \in[0,1) \\
\frac{1}{\sqrt{x}} & \text { if } x \in[1, \infty),
\end{array}\right.\right. \\
k y=\left\{\begin{array}{cl}
3 & \text { if } y \in[0,1) \\
\frac{1}{y} & \text { if } y \in[1, \infty) .
\end{array}\right.
\end{gathered}
$$

Let $\varphi(t)=2 t, p=2, a=1, \alpha=\frac{1}{4}$ and $\beta=\frac{3}{4}$, so, all conditions of Theorem 4 are satisfied and 1 is the unique common fixed point of mappings $f, g, h$ and $k$.

Before finishing our work, we present our fifth result.

Theorem 5 Let $\mathcal{X}$ be a nonempty set, $d$ a symmetric on $\mathcal{X}$. Let $f, g, h$ and $k$ be self-mappings of $\mathcal{X}$ such that

(5) $\int_{0}^{d(f x, g y)} \varphi(t) d t \leq \int_{0}^{g(d(h x, k y), d(f x, h x), d(g y, k y), d(f x, k y), d(g y, h x))} \varphi(t) d t$

for all $x, y$ in $\mathcal{X}$, where $\varphi$ is as in Theorem 2 and $g: \mathbb{R}_{+} \rightarrow \mathbb{R}_{+}$such that if $u \leq g(u, 0,0, u, u)$, then $u=0$. If $f$ and $h$ as well as $g$ and $k$ are owc, then $f$, $g, h$ and $k$ have a unique common fixed point.

Proof. By hypothesis, there exist elements $u$ and $v$ in $\mathcal{X}$ such that $f u=h u$ and $f h u=h f u ; g v=k v$ and $g k v=k g v$.

Suppose that $f u \neq g v$. Then, from (5),

$$
\begin{aligned}
& \int_{0}^{d(f u, g v)} \varphi(t) d t \leq \\
& \int_{0}^{g(d(h u, k v), d(f u, h u), d(g v, k v), d(f u, k v), d(g v, h u))} \varphi(t) d t \\
= & \int_{0}^{g(d(f u, g v), 0,0, d(f u, g v), d(g v, f u))} \varphi(t) d t
\end{aligned}
$$


which implies that $d(f u, g v)=0$. Hence $f u=g v$.

Now, suppose that $f^{2} u \neq f u$. Then, from (5),

$$
\begin{aligned}
& \int_{0}^{d\left(f^{2} u, f u\right)} \varphi(t) d t=\int_{0}^{d(f f u, g v)} \varphi(t) d t \\
\leq & \int_{0}^{g\left(d(h f u, k v), d\left(f^{2} u, h f u\right), d(g v, k v), d\left(f^{2} u, k v\right), d(g v, h f u)\right)} \varphi(t) d t \\
= & \int_{0}^{g\left(d\left(f^{2} u, f u\right), 0,0, d\left(f^{2} u, f u\right), d\left(f u, f^{2} u\right)\right)} \varphi(t) d t
\end{aligned}
$$

which implies that $f^{2} u=f u$.

Similarly, $g^{2} v=g v$. Hence $f u=g v$ is a common fixed point of mappings $f, g, h$ and $k$.

By inequality (5), the common fixed point is unique.

Remark 5 Letting $\varphi(t)=1$ in the above Theorem we obtain a generalization of Theorem 4 of [7].

We end our work by giving the last result.

Theorem 6 Let $\{f, h\}$ and $\{g, k\}$ be owc pairs of self-mappings of a nonempty set $\mathcal{X}$ endowed with a symmetric $d$. Consider the control function $\phi: \mathbb{R}_{+} \rightarrow$ $\mathbb{R}_{+}$such that $\phi(t)=0$ if and only if $t=0$. If

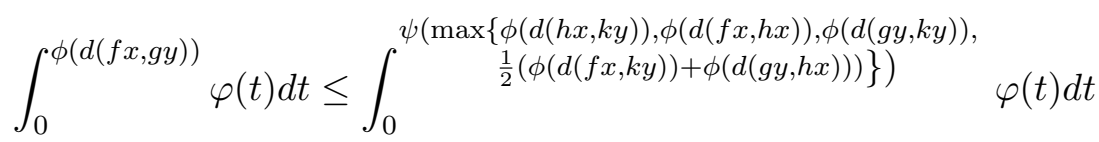

for all $x, y$ in $\mathcal{X}$, where $\psi: \mathbb{R}_{+} \rightarrow \mathbb{R}_{+}$such that $\psi(t)<t$ for each $t>0$ and $\varphi$ is as in Theorem 2. Then $f, g, h$ and $k$ have a unique common fixed point.

Proof. Since $\{f, h\}$ and $\{g, k\}$ are each owc, then, there exist $u$ and $v$ such that $f u=h u, f h u=h f u$ and $g v=k v, g k v=k g v$.

Suppose that $f u \neq g v$. Then, from (6),

$$
\begin{aligned}
& \int_{0}^{\phi(d(f u, g v))} \varphi(t) d t \\
\leq & \left.\left.\int_{0}^{\psi(\max \{\phi(d(h u, k v)), \phi(d(f u, h u)), \phi(d(g v, k v)),} \frac{1}{2}(\phi(d(f u, k v))+\phi(d(g v, h u)))\right\}\right) \\
= & \int_{0}^{\psi(\phi(d(f u, g v)))} \varphi(t) d t
\end{aligned}
$$


a contradiction. Therefore $\phi(d(f u, g v))=0$, which implies that $d(f u, g v)=0$; i.e., $f u=g v$.

Similarly, we can prove that $f^{2} u=f u$ and $g^{2} v=g v$ by using condition (6) which gives also uniqueness.

Remark 6 Theorem 5 of [7] is a special case of Theorem 6 with $\varphi(t)=1$.

Example 6 Consider the set $\mathcal{X}=[0, \infty)$ with the symmetric $d(x, y)=(x-$ $y)^{2}$. Define

$$
\begin{gathered}
f x=g y=\left\{\begin{array}{ll}
\frac{1}{2} & \text { if } x, y \in[0,1) \\
1 & \text { if } x, y \in[1, \infty),
\end{array} \quad h x=\left\{\begin{array}{cc}
2 & \text { if } x \in[0,1) \\
\frac{1}{x^{2}} & \text { if } x \in[1, \infty),
\end{array}\right.\right. \\
k y= \begin{cases}2 & \text { if } y \in[0,1) \\
\frac{1}{y} & \text { if } y \in[1, \infty) .\end{cases}
\end{gathered}
$$

Let $\varphi(t)=2 t, \phi(t)=t$ and $\psi(t)=\frac{t}{2}$, so, all conditions of Theorem 6 are satisfied and 1 is the unique common fixed point of mappings $f, g, h$ and $k$.

\section{Conclusion}

Our above results generalize theorems given in [2], [3], [7], [8], [9], [13], [14], [15], [16], [17], [18] and others since the notion of occasionally weakly compatible mappings is the most general concept among all the commutativity concepts. We further add that we did not require continuity, again we used the general contractive condition (1) in Theorem 1 which is more general than all inequalities. Also we deleted some assumptions imposed on functions $F$, $\varphi, \phi, g$ and $\psi$, on the metric space, on the constants and on the mappings, then, every theorem involving two or four mappings becomes a special case of one of our theorems.

Acknowledgements. The author is highly thankful to the anonymous referee for his/her appreciation, valuable comments, and suggestions. 


\section{References}

[1] M. A. Al-Thagafi, N. Shahzad, Generalized I-nonexpansive selfmaps and invariant approximations, Acta Math. Sin. (Engl. Ser.), vol. 24, no. 5, 2008, 867-876.

[2] H. Bouhadjera, General common fixed point theorems for compatible mappings of type $(C)$, Sarajevo J. Math., vol. 14, no. 2, 2005, 261-270.

[3] A. Branciari, A fixed point theorem for mappings satisfying a general contractive condition of integral type, Int. J. Math. Math. Sci., vol. 29, no. $9,2002,531-536$.

[4] G. Jungck, Compatible mappings and common fixed points, Internat. J. Math. Math. Sci., vol. 9, no. 4, 1986, 771-779.

[5] G. Jungck, Common fixed points for noncontinuous nonself maps on nonmetric spaces, Far East J. Math. Sci., vol. 4, no. 2, 1996, 199-215.

[6] G. Jungck, P. P. Murthy, Y. J. Cho, Compatible mappings of type $(A)$ and common fixed points, Math. Japon., vol. 38, no. 2, 1993, 381-390.

[7] G. Jungck, B. E. Rhoades, Fixed point theorems for occasionally weakly compatible mappings, Fixed Point Theory, vol. 7, no. 2, 2006, 287-296.

[8] J. K. Kohli, S. Vashistha, Common fixed point theorems for compatible and weakly compatible mappings satisfying general contractive type conditions, Stud. Cercet. Ştiinţ. Ser. Mat. Univ. Bacău, vol. 16, 2006, 33-41.

[9] S. Kumar, R. Chugh, R. Kumar, Fixed point theorem for compatible mappings satisfying a contractive condition of integral type, Soochow J. Math., vol. 33, no. 2, 2007, 181-185.

[10] H. K. Pathak, Y. J. Cho, S. M. Kang, B. S. Lee, Fixed point theorems for compatible mappings of type $(P)$ and applications to dynamic programming, Matematiche (Catania), vol. 50, no. 1, 1995, 15-33. 
[11] H. K. Pathak, Y. J. Cho, S. M. Kang, B. Madharia, Compatible mappings of type $(C)$ and common fixed point theorems of Greguš type, Demonstratio Math., vol. 31, no. 3, 1998, 499-518.

[12] H. K. Pathak, M. S. Khan, Compatible mappings of type $(B)$ and common fixed point theorems of Greguš type, Czechoslovak Math. J., vol. 45, no. 4, 1995, 685-698.

[13] H. K. Pathak, R. Tiwari, M. S. Khan, A common fixed point theorem satisfying integral type implicit relations, Appl. Math. E-Notes, vol. 7, 2007, 222-228.

[14] V. Popa, Some fixed point theorems for compatible mappings satisfying an implicit relation, Demonstratio Math., vol. 32, no. 1, 1999, 157-163.

[15] V. Popa, Common fixed point theorems for compatible mappings of type (A) satisfying an implicit relation, Stud. Cercet. Ştiinţ. Ser. Mat. Univ. Bacău, vol. 9, 1999, 165-172.

[16] V. Popa, A general fixed point theorem for compatible mappings of type $(P)$, Stud. Cercet. Ştiinţ. Ser. Mat. Univ. Bacău, vol. 11, 2001, 153-157.

[17] V. Popa, A general fixed point theorem for four weakly compatible mappings satisfying an implicit relation, Filomat, vol. 19, 2005, 45-51.

[18] B. E. Rhoades, Two fixed-point theorems for mappings satisfying a general contractive condition of integral type, Int. J. Math. Math. Sci., vol. 63, 2003, 4007-4013.

[19] S. Sessa, On a weak commutativity condition of mappings in fixed point considerations, Publ. Inst. Math., vol. 32, no. 46, 1982, 149-153.

\section{Hakima Bouhadjera}

Badji Mokhtar-Annaba University

Faculty of Sciences

Department of Mathematics

Laboratory of Applied Mathematics, P.O. Box 12, 23000 Annaba, Algeria

e-mail: b_hakima2000@yahoo.fr 